\title{
L'impact sur l'Italie des débats français sur la langue au milieu $\mathrm{du} \mathrm{XVI}^{\mathrm{e}}$ siècle
}

\author{
FRANÇOIS PARÉ
}

Summary: While Sperone Speroni's Dialogo delle lingue is usually considered the primary source of Joachim Du Bellay's Deffence et Illustration de la langue françoyse, the impact of French linguistic thought on late sixteenth-century Italy is far more difficult to ascertain. In opposition to French, which quickly constructs itself as the instrument of the rising hegemonic French state, Italian, widely used around the Mediterranean as a lingua franca, does not conform to a strict nationalistic program. On all fronts a language in contact, Italian, as portrayed in the Dialogo delle lingue, must be seen in a context of a prevalent cultural heterogeneity.

I es constructions mentales, par lesquelles nous tentons le plus souvent de Lcomprendre l'histoire de l'Europe selon le vecteur spatial sud-nord, accordent à l'Italie et à certaines des cultures méditerranéennes une valeur exemplaire dans le développement des entités nationales, pour ce qui est de la partie plus septentrionale du continent. La transformation de l'histoire culturelle européenne, celle de la France, de l'Allemagne et de l'Angleterre en particulier, à partir du paradigme de l'Antiquité classique, a suscité, en effet, un puissant imaginaire nostalgique que la pensée déconstructionniste, sous l'impulsion des théories de Michel Foucault et de Jacques Derrida, n'a guère encore déstabilisé. Cette organisation de l'histoire de la transmission des idées dans le temps et dans l'espace s'est donc trouvée renforcée, depuis plusieurs siècles, par le rôle fondateur attribué à la mémoire de l'Antiquité gréco-romaine, minutieusement cultivée d'abord par les lettrés et ensuite, jusqu'à ce jour, par l'université. Comme l'écrivait, de façon symptomatique, Enea Balmas dans son manuel bien connu d'histoire littéraire de la Renaissance, «L'Europe, c'est avant tout, dans la perspective qui nous intéresse, 
l'Italie et l'Espagne, les seuls grands pays d'Occident qui possèdent déjà, au $\mathrm{XVI}^{\mathrm{e}}$ siècle, une langue et une culture en mesure d'exercer une influence et de proposer des modèles ${ }^{1}$. Pour cet historien, l'Europe renaissante, celle du nord, se serait donc alimentée aux cultures du sud, dont elle se serait appropriée l'histoire et les textes. Cette ligne de transmission continue de nourrir l'imaginaire critique en dépit de ruptures successives importantes qui auraient dû permettre d'envisager les espaces culturels et linguistiques européens selon d'autres paramètres.

Il importe de souligner, avant d'aller plus loin, que l'Italie, l'Espagne et la Grèce, entre autres, ont profondément ressenti les contrecoups de cette politique bipolaire des espaces culturels européens. Dès le début du XVI ${ }^{\mathrm{e}}$ siècle, un profond travail de substitution et de récupération est en jeu, qui vise à priver de légitimité les langues et les cultures du pourtour méditerranéen. Reconnues pour leur passé glorieux, les nations du sud se sont vues du même coup dépossédées du présent et exclues des enjeux contemporains, liés largement à la diffusion du livre imprimé et à l'expansion coloniale. Ces cultures, à la fois glorifiées et anéanties, mettront des centaines d'années à se remettre de cette spoliation qui consacrait leur centralité historique dans l'espace européen en même temps que leur mise à l'écart du présent et de l'avenir de cet espace même.

Tout se passe donc, au sortir du Moyen Âge, comme si les cultures et les langues du sud de l'Europe avaient donné naissance à celles du nord, alors que se produisait un immense effort de traduction et d'adaptation qui constituerait éventuellement ce qu'on appelle la Renaissance. Si elle a d'abord appartenu à Florence, à Gênes et à Venise, cette Renaissance a aussi signé la fin de l'hégémonie réelle de ces états et leur marginalisation définitive. Je ne pense pas que nous puissions remettre en cause cette géographie singulière des cultures européennes. Nous aboutirions sans doute à une aporie de l'histoire elle-même, puisqu'il est aujourd'hui extrêmement difficile d'inverser des tracés et des trajectoires emblématiques, sans lesquels les contacts constants qui ont enrichi au cours des âges les cultures de l'Europe ne peuvent être décrits. Reste que l'enjeu d'une telle substitution, survenue alors que se profilaient les empires conjugués du livre et de la colonisation, ne saurait être ignoré 2 . D'abord centrales dans le développement de la pensée européenne, les cultures méditerranéennes ont été ensuite largement reléguées à la périphérie de l'histoire, puisque le sud apparaissait comme le double ferment d'une naissance extraordinaire à la culture et le signe avant-coureur de sa déchéance inévitable. Toutes les cultures de la Méditerranée, de la Grèce à l'Espagne et de Chypre aux Baléares, ressentent encore aujourd'hui avec force la grandeur et le déclin qui coexistent en elles dans ces lectures particulières de l'histoire depuis la Renaissance. Déjà, 
parmi d'autres, Joachim Du Bellay avait saisi, lors de son voyage à Rome, l'ampleur décisive de ce paradoxe. Les Regrets et surtout les Antiquitez de Rome soulignaient la nécessité de transformer les « ruines » linguistiques et morales des cultures méditerranéennes en un terreau de renouvellement pour les nations du nord.

Bien que ces formes soient encore très présentes dans le discours critique actuel sur la Renaissance, serait-il possible un instant de penser les choses différemment? Quel renversement des perspectives peut-on encore imaginer? Si, dans Devant le temps, Georges Didi-Huberman nous encourage à faire usage de l'anachronisme dans l'étude des phénomènes historiques, on pourrait penser qu'une inversion de l'espace où se sont manifestés à une certaine époque les échanges culturels et linguistiques, qu'une sorte d'anatopisme stratégique pourrait montrer la voie vers une réflexion nouvelle sur la Renaissance ${ }^{3}$. Comme nous le verrons en ces lignes, un tel renversement n'est guère facile à élaborer, puisque la documentation existante et les conventions historiques, mises en place dès le milieu du XVI ${ }^{\mathrm{e}}$ siècle tendent à faire silence sur ses conditions de possibilité. Je me contenterai ici de ne considérer qu'un seul aspect, celui des rapports interlinguistiques, en sachant bien que la question des langues en contact, telle que la définissent Henri Boyer ${ }^{4}$, Joshua A. Fishman ${ }^{5}$ et d'autres théoriciens actuels, s'inscrit forcément dans une conception plus large des corpus historiques et des idéologies économiques. Ce qui est certain, c'est que ces phénomènes interlinguistiques (entre l'italien et le français, entre le turc et l'italien, entre l'allemand et les langues slaves), déjà bien visibles dans l'Europe de la Renaissance, constituent les signes avant-coureurs d'une remarquable expansion des zones de contacts et de contamination entre les langues et les cultures dont nous voyons sans doute le plein aboutissement à notre époque.

\section{Mémoire et pluralité chez Sperone Speroni}

Quel a été l'impact transalpin des théories françaises sur la langue au milieu $\mathrm{du} \mathrm{XVI}^{\mathrm{e}}$ siècle ? Ces théories, visant à faire du français une langue littéraire et un symbole du renouveau politique en France, ont-elles traversé à leur tour les frontières de l'Italie, pour venir renforcer le vif débat sur la codification et les usages poétiques et politiques de la langue italienne, débat entrepris dès le milieu du XVe siècle en Toscane et dans la République de Venise notamment? Il convient d'attirer l'attention, d'abord, sur la période qui précède la parution à Paris de la Deffence et Illustration de la langue françoyse (1549) de Joachim Du Bellay, un ouvrage absolument crucial sur les plans institutionnel et politique, que son auteur avait emprunté, sans toutefois reconnaître sa dette, au Dialogo delle lingue (1542) de Sperone 
Speroni ${ }^{6}$. Pour comprendre la place qu'a pu occuper en retour La Deffence et Illustration de la langue françoyse dans les différents espaces culturels et linguistiques de l'Italie, il faut rappeler la forte influence du livre et surtout du personnage de Speroni dans la seconde moitié du siècle. En s'appropriant un discours de la modernité «sudiste », Du Bellay déplace considérablement les enjeux liés à la politique et à la poétique de la langue vernaculaire, tels que les avait proposés Speroni, afin de souligner avant tout l'hybridité « moderne » du matériau linguistique, l'instabilité des langues dans l'histoire et les forces symbiotiques qui sont à l'œuvre dans les langues et qui rendent possibles leurs naissances successives.

Au-delà des ouvrages philosophiques dont il a été l'auteur, c'est par sa personnalité publique que Speroni joue un rôle central dans la promotion de la langue vernaculaire italienne, codifiée sur le plan du lexique et grammaticalisée sur le plan des structures phrastiques. Le Dialogo delle lingue avait certainement la prétention de rassembler en un seul livre l'ensemble des opinions contemporaines sur la langue en Italie, de Bembo à Sannazaro, en passant par Cortegiano et Lazaro. Ces théoriciens toscans, tous plus ou moins contemporains de Speroni, jouent à la fois leur propre rôle et celui de personnages fictifs dans le dialogue qui s'engage sur la diversité linguistique et la nécessité d'une norme commune. Cependant, chez Speroni, le Padouan, nulle position hégémonique sur la langue n'émerge. Chacun des linguistes invités s'obstine, se braque, sans pour autant que le dialogue s'enraie. Les échanges sont vigoureux. Le ton monte. Cortegiano, qui joue souvent le rôle du modérateur, appelle chacun au calme. C'est que les enjeux de la langue sont cruciaux. De cela, tous les «personnages » du Dialogo delle lingue sont absolument convaincus. Pour certains, la supériorité des langues de l'Antiquité reste incontestable, dans la mesure où la « pauvre Italie » n'arrivera jamais à faire valoir la valeur littéraire du vernaculaire toscan. Pour d'autres, ce vernaculaire manifeste la vulgarité même de l'époque présente, incapable de s'inspirer des richesses linguistiques du passé et surtout coupée de la bontà, si présente, à leurs yeux, dans la tradition classique. À la fin, le texte de Speroni reste étonnamment pluriel dans son accueil des divergences d'opinion. Par son habile mise en scène des perspectives opposées sur l'avenir du toscan, Speroni met en lumière la rencontre extraordinaire de la politique linguistique (la lingua) et de la valeur morale (la bontà), telles que celles-ci se résument dans l'adoption d'une langue commune.

Ainsi, plus que l'auteur d'un assez curieux dialogue des langues, une sorte de table ronde avant l'heure, Speroni apparaît comme l'héritier de toute une génération de poètes et de lettrés qui avaient fait de la promotion de la langue vernaculaire le cœur même de leur écriture et de leur engagement politique. Au moment où Joachim Du Bellay «emprunte » le texte du 
Dialogo delle lingue, pour en faire l'argument principal de sa Deffence et Illustration de la langue françoyse, la réputation de Speroni dépasse largement sa région d'origine. Cette renommée ne cessera de s'accroître jusqu'à sa mort à Padoue en 1588. En 1570, Le Tasse lui confie d'ailleurs la relecture de son poème de la Jérusalem, œuvre majeure qu'il s'apprête à publier et pour laquelle il sollicite l'approbation du grand linguiste. Âgé de 77 ans, Speroni s'exécute et corrige le manuscrit.

Les écrits de Speroni et le Dialogo delle lingue, en particulier, transcendent donc toute une époque et toute une problématique pan-européenne, liée aux représentations des langues vernaculaires et des valeurs de renouvellement qu'elles véhiculaient. Il n'est pas étonnant que Joachim Du Bellay ait cherché à s'alimenter à cette œuvre exceptionnelle qui devait servir de phare à quiconque chercherait à élaborer une poétique/politique de la modernité linguistique et culturelle. Cependant, Du Bellay transformera profondément le ton de l'original italien, parce que la structure ouverte et plurielle de l'œuvre de Speroni ne pouvait guère convenir aux modèles politiques qui allaient éventuellement consolider l'idée de nation en France au début du XVII ${ }^{\mathrm{e}}$ siècle.

Certes, chez Speroni, le renvoi au concept fondamental de la mémoire culturelle était largement présent. Tous les interlocuteurs du Dialogo font appel à l'héritage des langues grecque et latine ; nombreux sont ceux, comme le personnage de Lazaro, qui proclament la permanence de cette transmission. En effet, n'est-ce pas là le principal enjeu : l'accès du monde contemporain à l'éternité proclamée de cette mémoire commune, dont l'histoire confirmait la place « substituante », selon le mot attribué par Speroni à Cortegiano ? Cette mémoire détermine toute discussion sur la langue vernaculaire, puisque les nations «modernes » cherchent justement à se substituer (sottoponere) à celles du passé gréco-romain. Le latin, on l'a souvent dit, ne semble-t-il pas faire de ses locuteurs des participants à la mémoire civilisatrice, affirmant par cette langue même sa pérennité absolue, son accès à une culture de l'immobilité, sereine et explicite ? Cette mémoire peut-elle aujourd'hui s'apprendre ? Là-dessus, les avis du Dialogo delle lingue sont partagés. Si chacun, en fin de compte, croit irréalisable l'apprentissage du latin et du grec par les modernes, c'est parce que la conscience aiguë du temps fracture la mémoire et rend impossible l'imaginaire unifié du passé.

C'est pourquoi il faut s'interroger sur les formes caduques, propres aux langues vernaculaires (croit-on), formes déchues que ces langues charrient inlassablement. Sans fixité aucune et soumises aux caprices de tout un chacun, elles semblent incapables d'avoir prise sur l'éternité. Comment la culture saurait-elle se transmettre à l'humanité future par de tels instruments 
linguistiques, marqués par le manque et l'incertitude ? Car, ni pour Speroni, ni pour Du Bellay, la langue ne s'offre dans sa plénitude affirmée. Elle est toujours frappée d'une carence que les contemporains cherchent à combler de multiples manières, tant par l'emprunt que par le néologisme. Déjà, chez les différents interlocuteurs du dialogue speronien, nous retrouvons une idée fondamentale de la Deffence et Illustration de la langue françoyse, à savoir que la langue vernaculaire, en dépit de l'égalité présumée des codes linguistiques, dont se réclame Du Bellay, ne peut être que porteuse de dégénérescence et d'oubli. Seule une intervention poétique dans le jeu politique de la langue permettrait peut-être de renverser ces conditions du déclin. C'est pourquoi la poésie fera de l'oubli, intrinsèque à la langue, son principe et sa méthode.

Les enjeux, pour Speroni comme pour Du Bellay, sont énormes. C'est du moins leur conviction, au moment où le poète français reprend pour lui-même les arguments du linguiste italien. Sans doute, il pourrait advenir que les langues vernaculaires de la modernité, loin d'être porteuses d'une moralité nouvelle, soient au contraire les signes répétés de la mort à venir de toutes les civilisations, ou en tout cas de leur enfoncement dans l'oubli, leur exclusion subtile, hors de la mémoire. Si de telles angoisses nous paraissent aujourd'hui assez prévisibles (et encore !), il ne faut pas croire qu'elles l'étaient nécessairement au milieu du XVIe siècle. En effet, tout le débat sur la valeur des langues vernaculaires reposait sur une surdétermination du concept de déclin dans la culture de la Renaissance. Là où Speroni se contentait parfois d'affirmer la supériorité du toscan sur les autres langues écrites de la péninsule italienne, Du Bellay, quant à lui, comprend plus que jamais la portée politique pour l'époque présente du débat sur la langue vernaculaire.

La structure dialogique et la multiplicité des voix qui opèrent dans le Dialogo delle lingue de Speroni confèrent, par ailleurs, à cette œuvre une dimension plurielle assez frappante. Dans ce texte, où alternent les voix parfois discordantes d'interlocuteurs réels, le lecteur a du mal à trouver l'unité du propos. Une impression de digression, notée par les personnages eux-mêmes, persiste au-delà de la lecture. Bien que la présence auctoriale soit bien sentie, surtout vers la fin du dialogue, nulle conclusion ne vient clore le traité. Aucun des interlocuteurs ne semble l'emporter; il faudra mettre en relation les différentes pétitions de principe, chercher une part commune que le dialogue ne fait qu'interroger jusqu'à la dernière réplique. Cette caractéristique du texte speronien est fondamentale.

Or, la pluralité est entièrement perdue dans la lecture et la traduction que fait Joachim Du Bellay du Dialogo delle lingue. Dans la Deffence et Illustration de la langue françoyse, en effet, une seule voix auctoriale prend 
la responsabilité du discours moral et poétique sur la langue. Curieusement, là où l'assemblée des linguistes avait échoué, c'est bien le poète qui cherchera à imposer sa loi. Si une dissension semble possible à certains moments, surtout dans les premières pages du manifeste, elle est vite résorbée au sein du projet politique qui recoupe toute l'œuvre. Du Bellay entend prendre part explicitement à la construction d'une nation française forte et éclairée, dont l'affirmation reposera sur une véritable poétique de la mémoire. Cette intervention appuyée du poétique, à l'image de la royauté dont Du Bellay se fait le défenseur, ne pouvait s'inspirer entièrement du modèle offert par l'œuvre de Speroni, beaucoup trop respectueux des différences régionales et individuelles. Du Bellay ne préservera de Speroni que l'argument central de la mémoire. À l'échange des idées, à la «table ronde»speronienne, Du Bellay substitue le manifeste poétique, qui cherchera à refonder dans l'Un l'autorité toujours lacunaire, toujours perdue de la langue vernaculaire.

\section{De Du Bellay à Speroni : improbable effet de retour}

De l'aire italienne à l'aire française, la «traduction » du texte de Speroni par Joachim Du Bellay a opéré un déplacement définitif qui rendra irrecevable, en retour, la lecture du manifeste français après le milieu du XVI siècle. Les effets d'appropriation sont d'autant plus importants que la Deffence et Illustration de la langue françoyse est assez rapidement récupérée par le milieu lettré en France. Cette réception tendra à insérer la question de la langue vernaculaire dans un débat qui paraît essentiellement français. Si des échanges transfrontaliers entre l'Italie et la France continuent de se faire après 1550, ils sont toujours largement dans l'axe sud-nord, comme le montrent certaines traductions, dont celle de Speroni par Gruget, et l'ensemble de la production lyonnaise dans la seconde moitié du siècle. En Italie, le manifeste linguistique de Du Bellay, en autant qu'on puisse en retrouver la trace, ne circule guère, bien que l'auteur des Regrets ait été connu de plusieurs à Rome et dans le nord de l'Italie. Ce sont plutôt les textes italiens de la première heure - ceux de Speroni, Miachiavel, Bembo - que l'on reproduit. L'institution littéraire naissante en tirera des pages célèbres dont la culture italienne ne se départira plus. Ce sont elles que l'on retrouve encore aujourd'hui sur les nombreux sites Internet. Il y a donc peu de traces du référent français. Du Bellay s'est lui-même rendu dans cette Italie à laquelle il vouait une grande admiration, mais son œuvre ne l'a pas suivi pour autant, car elle ne correspondait pas, dans les transformations qu' elle avait fait subir au Dialogo delle lingue, à la conjoncture linguistique italienne, beaucoup plus diffuse et surtout plus divergente sur le plan politique. 
Speroni meurt en 1588. Il est le dernier survivant d'un magnifique débat sur le modèle toscan et l'avenir de la langue italienne, auquel avaient contribué des dizaines de ses contemporains. Pourquoi la pensée française de la langue, arrimée à la construction d'une monarchie forte et d'une nation pérenne, n'a-t-elle pas eu d'écho véritable dans les différentes régions de l'Italie ? Pour comprendre les enjeux de la situation linguistique dans la péninsule italienne, il faut rappeler que l'Italie reste encore, au milieu du $\mathrm{XVI}^{\mathrm{e}}$ siècle, un espace géographique très diversifié sur le plan linguistique ${ }^{7}$. Dans ce contexte, le toscan ne pouvait aspirer à occuper la place hégémonique que le français parviendra assez rapidement à acquérir au-delà des Alpes. Il ne pouvait servir que d'instrument de médiation, de lingua franca, dans une aire culturelle largement excentrée.

Il faut dire qu'en dépit d'efforts assez systématiques après le milieu du $\mathrm{XV}^{\mathrm{e}}$ siècle ni la langue française ni la langue italienne (ni ses multiples variétés dialectales) ne sont véritablement codifiées. Si, en France, le projet de grammaticalisation de la langue et la standardisation des orthographes recueillaient un appui sensible chez les lettrés et dans la classe dirigeante, la même démarche échappait largement au domaine italien, déchiré par des rivalités persistantes entre les régions politiques et linguistiques ${ }^{8}$. En outre, des formes relativement variables de la langue italienne servaient depuis longtemps au commerce intrapéninsulaire. Par elle, les échanges semblaient possibles en dépit des frontières politiques. Cette langue circulait dans de nombreuses régions du pourtour méditerranéen : «Lingua di servizio », rappelle l'historien Francisco Bruni, « limitada negli scopi e nel ventaglio communicativo, la lingua franca sarà stata certo mutevole nel tempo e nello spazio, essendo privata di qualunque codifica ${ }^{9}$. Il est certain que bon nombre de commerçants voyageurs, de Rome jusqu'à Alger, de Lyon jusqu'à Ragusa (Dubrovnik), avaient appris l'italien, dans la mesure où l'usage d'une langue commune, bien que fort peu codifiée, leur paraissait nécessaire à la multiplication des marchés. Culturellement et politiquement, l'Italie du $\mathrm{XVI}^{\mathrm{e}}$ siècle ne se conçoit donc pas nécessairement comme une nation en devenir, mais plutôt comme le centre d'une zone d'influence économique qui excède largement ses frontières et que le nationalisme naissant ne pouvait satisfaire entièrement ${ }^{10}$.

De plus, sur le plan strictement linguistique, la langue toscane, que discutaient dans l'abstrait les personnages du Dialogo delle lingue, restait soumise assurément à d'énormes pressions, la pluralité de ses locuteurs rendant ses structures instables et changeantes. L'italien (toscan ou vénitien), dont faisait usage un Lyonnais, un Croate ou un Piémontais, se présentait sans doute beaucoup plus comme une "langue en contact », marquée par l'hétérogénéité des formes, imposée par la grande diversité des 
locuteurs. Certes, de la même manière, le français a dû servir de « langue seconde » à une grande partie de la population, habitant le territoire français à l'époque où Du Bellay en entreprend la « défense ». Mais, par son rayonnement transfrontalier, la langue italienne se construit justement comme le code emblématique de la diversité méditerranéenne. Dans sa brève histoire de la langue italienne, Bruni fait encore remarquer que, partout sur le territoire et surtout dans la partie orientale de la péninsule, la langue italienne a dû coexister avec d'autres langues, dont le provençal, le dalmate, les dialectes germaniques, le slovène et, plus au sud, le grec et le turc. Sur les rives de l'Adriatique, dans l'aire d'influence de Venise, il est fréquent d'observer que les hommes échangent entre eux en italien, tandis que les femmes, ne comprenant pas cette langue, continuent de converser en dalmate ou en slovène.

À Venise même, le statut de la langue italienne reste problématique jusqu' au XVII' siècle au moins. À l'intérieur comme à l'extérieur de l'Italie, les positions centralisatrices, exprimées par Du Bellay dans la Deffence et Illustration de la langue françoyse, ne paraissent aucunement recevables pour la majorité des lettrés, à l'exception de l'élite toscane. En 1607, Francesco Sansovino note encore que la majorité des Vénitiens ne considèrent pas leur langue comme une version dialectale de l'italien des Florentins ou des Génois. Machiavel, on le sait, s'était attardé à ces vacillements de l'identité linguistique dans son Discorso intorno alla nostra lingua. En cherchant à accommoder la diversité régionale dans le fonds commun de la langue péninsulaire, Machiavel s'était trouvé à anticiper, en quelque sorte, les arguments de Du Bellay. En effet, tout code linguistique serait, selon Machiavel, le fruit de rencontres aléatoires entre les codes et de leur imbrication progressive. Toute langue est autre par son histoire même : "Perché non si puo trovare una lingua che parli ogni cosa per sé senza haverne accattato da altri ; perché, nel conversare gl'huomini di varie provincie insieme, prendono de' motti l'uno dell'altro ${ }^{11}$. Chez Du Bellay, une distinction importante s'impose : si la langue française, principe de la nation en émergence, peut s'appuyer sur une hybridité historique, celle de l'héritage gréco-romain, elle ne peut guère, par contre, admettre l'apport des langues et dialectes régionaux de la France contemporaine. Pour Machiavel, la diversité même du territoire linguistique italien renforce la position de cette langue comme le lieu d'un compromis nécessaire au bien commun.

La culture italienne s'est longtemps inscrite, on le voit, dans une tout autre économie de la diversité linguistique. Le multilinguisme en vigueur dans l'aire méditerranéenne ne pouvait être absorbé par la promotion d'une langue uniformisée, structurée par son origine claire, comme le proposait Du Bellay dans son manifeste. Bien sûr, la Deffence et Illustration de la 
langue françoyse tient compte de façon remarquable de l'hybridité de toute langue. Toutefois, la perspective de Du Bellay reste entièrement diachronique. Alors que l'origine du français est le produit d'une rencontre historique (sinon mythique) entre le sud et le nord, cette hétérogénéité foncière s'effacerait aujourd'hui sous l'influence d'une synthèse poétique et surtout politique. Le facteur étymologique, extrêmement important dans le discours de La Pléiade, prendra un caractère quasi ontologique. La racine du mot, au cœur même du présent, deviendra le fantasme d'une certaine permanence au sein de la langue fragmentée et trafiquée par le temps. En Italie, il est vrai que, dès le XIII ${ }^{e}$ siècle, la culture toscane repose sur la promotion d'une langue littéraire originale, détachée de ses contingences variables. Mais la langue vernaculaire, telle qu'elle se construit dans la péninsule italienne à l'encontre même de la visée toscane, se définit précisément par sa mutabilité et son absence de frontières ontologiques.

Ces remarques restent encore conjecturales. Car l'on n'a pas assez étudié la question des contacts et des échanges linguistiques et culturels dans l'espace européen à la Renaissance. Une telle étude, entreprise sur plusieurs fronts, nous amènerait à repenser, sur le mode de l'hybridité, les anciennes polarisations géographiques et la formation des nations modernes. Il faudrait pour être juste suivre la circulation des idées et des comportements linguistiques dans l'ensemble de la Méditerranée. Les langues de l'Europe, en dépit des théories uniformisantes dont elles ont fait l'objet, sont des lieux de frontières, riches de la multiplicité des codes en constant chevauchement et en constante irradiation. Ce foisonnement dont certaines cités renaissantes ont été l'exemple (Lyon, Venise, Ragusa, Gênes) et dont le Dialogo delle lingue de Speroni rendait compte, détermine l'écart qui sépare les espoirs politiques de la culture lettrée et les contraintes quotidiennes de populations diverses en interaction les unes avec les autres. En France, il aura fallu les efforts de tout un système politique et poétique autoritaire, sur plusieurs siècles, pour arriver à réduire et appauvrir cet univers linguistique en mutation. Il nous incombe d'en restaurer la validité historique et culturelle.

\section{University of Waterloo}

\section{Notes}

1. Enea Balmas, Littérature française. La Renaissance. II - 1548-1570 (Paris, Arthaud, 1974), p. 55.

2. C'est dans l'ouvrage important de Walter Mignolo que l'on trouve les paragraphes les plus intéressants sur cette conjoncture qui lie définitivement la diffusion de l'imprimé au colonialisme européen. Voir Walter Mignolo, The Darker Side of the Renaissance : Literacy, Territoriality and Colonization (Ann Arbor, University of Michigan Press, 1995), 
chap. 2, p. 69-122 ; aussi François Paré, « Le déclin de Gutenberg. Écriture imprimée et corruption des codes dialogiques à la fin de la Renaissance », dans La Renaissance hier et aujourd'hui, éd. Guy Poirier (Paris, L'Harmattan ; Québec, Presses de l'Université Laval, 2002), p. 67-87.

3. Georges Didi-Huberman, Devant le temps. Histoire de l'art et anachronisme des images (Paris, Minuit, 2000).

4. Henri Boyer, Introduction à la sociolinguistique (Paris, Dunod, 2001).

5. Joshua A. Fishman, Language and Ethnicity in Minority Sociolinguistic Perspective (Clevedon, Multilingual Matters, 1989).

6. Le texte original du Dialogo delle lingue est disponible sur Internet : www.intratext. com/IXT/ITA1578/_P1.htm. Il faut noter également que la première traduction officielle de l'ouvrage de Speroni, celle de Claude Gruget en 1551, est disponible grâce aux efforts du collectif EPISTEMON : www.cesr.univtours.fr/EPISTEMON/cornucopie/NOTISpero.asp.

7. Voir Francesco Bruni, L'italiano nelle regioni : testi e documenti (Turin, UTET, 1994).

8. L'ensemble des livres d'histoire de la langue italienne font foi de ces rivalités, alors qu'un très grand nombre d'ouvrages français s'attardent aux perceptions régionales, ayant eu cours à la même époque. Voir, entre autres, Riccardo Tesi, Storia della lingua comune dalle origini al Rinascimento (Rome, Edizione Laterza, 2001).

9. Francisco Bruni, « Storia della lingua italiana », disponible sur Internet : www.italica. rai.it/principal/lingua/bruni/lezioni.htm.

10. Voir David Abulaifa, Mediterranean Encounters, Economic, Religious, Political, 11001550 (Burlington, VT, Ashgate, 2000).

11. Niccolò Machiavelli, Discorso o Dialogo intorno alla nostra lingua, éd. Bortolo Tommasso Sozzi (Turin, Einaudi, 1976). Voir le commentaire de Ornella Castellani Polidori, Nuove riflessioni sul Discorso o Dialogo intorno alla nostra lingua di Niccolò Machiavelli (Rome, Salerno Editrice, 1981). Le texte du Discorso intorno alla nostra lingua est disponible sur de nombreux sites Internet, dont : http://digilander.libero.it. 